\title{
Premessa
}

\section{LE VISIONI DI ILDEGARDA DI BINGEN}

HILDEGARD VON BINGEN'S VISIONS

Ferruccio Bertin Universidad de Genova, Italia

RiAssunto

Abstract:

Excursus sulla vita di Santa Ildegarda, una Outline about the life of Saint Hildegard, a profetessa la cui esistenza venne caratterizzata prophetess whose existence was characterized da peculiari visioni. Fu badessa in un'epoca by peculiar visions. She was a prioress during (XII secolo) in cui essere donna di chiesa era an age (11th century) when being a woman malvisto e la prima cristiana a considerare la from church was considered badly. She was femminilita intesa come tale. Nonostante gli the first who considered femininity that way. studi scarsi, fu in grado di comprendere testi She was able to understand texts from the

biblici. $\quad$ Bible, besides her poor studies.

Parole chiave:

disione, Dio, donna, uomini, Hildegard, vision, God, woman, men monastero
Io concordo pienamente con quanto afferma Barbara Newmann (1987) alle pp.XVI e XVII dell'Introduzione al volume Sister of Wisdom. St.Hildegard's Theology of the Feminine e cioè che: "L'importanza della sua posizione politica è stata oscurata dal fatto che ci si è concentrati sul suo misticismo, benché sotto questo rispetto ella abbia anticipato le successive 'visionarie' politiche, come Caterina da Siena e Brigida di Svezia.

Il suo predicare apocalittico - l'unico aspetto del suo lavoro che abbia largamente influenzato i tre secoli successivi - era strettamente connesso con il suo programma di riforma della Chiesa. Da una prospettiva diversa, si potrebbe collocare Ildegarda tra quegli autori altamente produttivi dell'inizio del XII secolo - come Ugo da S.Vittore, Ruperto di Deutz, Onorio di Regensburg - che solitamente sono definiti 'prescolastici'.

Tutti costoro scrissero copiosamente e, come Ildegarda, tutti quanti ascesero la scala del pensiero cristiano, offrendo una miscela di commenti alla Bibbia, ammaestrament morali e spirituali e istruzioni dogmatiche in forme che svariano dall'opuscolo all'enciclopedia.

Le opere di Ildegarda, nonostante le peculiarità del loro stile, presentano un corpus di ammaestramenti essenzialmente conservativi che si rifanno a questi più anziani contemporanei sia per il fine, sia per gli insegnamenti specifici. Non si deve permettere alla sbalorditiva originalità delle sue formulazioni di oscurare la fondamentale ortodossia del suo classico approccio benedettino alla vita spirituale".

La Newmann, tuttavia, considera Ildegarda da un altro punto di vista, cercando di collocarla in una tradizione 'verticale', osservando alcune delle sue principali tematiche alla luce di quanto la studiosa definisce sapiential tradition. Con questa definizione ella si riferisce alla perenne scuola di pensiero cristiano, che si basa sulla scoperta e l'adorazione della 'sapienza divina' nell'opera di creazione e redenzione.

I teologi di questa scuola, la cui storia si estende dalla Chiesa delle origini al XII secolo, condividono la predilezione per alcune tematiche: la bellezza divina, l'aspetto femminile di Dio, l'assoluta predestinazione di Cristo e di Maria, l'ideale etico ed estetico della verginità e la speranza della redenzione del mondo.

Ildegarda rappresenta un centro ideale per uno studio di questo genere, perché ella fu non solo un'esponente del sapiential thought, ma anche una figura centrale per il suo sviluppo. Il suo sesso, naturalmente, non è fortuito. Possiamo (afferma ancora la Newmann) tranquillamente definire Ildegarda come la prima pensatrice cristiana a prendere in considerazione seria e positiva la femminilità intesa come tale e non 
puramente come le provocazioni da e per le donne in un mondo dominato dagli uomini.

Ma ora è giunto il momento di fornire qualche indicazione biografica sul nostro personaggio: Ildegarda (1098-1179) seppe come pochi in due millenni di storia riecheggiare le parole di S.Paolo, quando la Chiesa di Corinto lo costrinse a difendere le sue rivendicazioni apostoliche. Egli prese posizione contro le improbabili fondamenta sulla follia e sulla debolezza che costituivano un precedente che aveva messo alla prova i capi della Chiesa fino ad allora. Eppure ella ebbe il coraggio di confutare una famosa affermazione del santo, contenuta in Cor. 11,9: "L'uomo non è stato creato per la donna, ma la donna è stata creata per l'uomo", replicando con questa frase "La donna è stata creata per l'uomo, e l'uomo è stato foggiato per la donna, (F?hrk?tter, 1990: I 1, 12, 302-303). Ma tale vanto è doppiamente paradossale, poiché questa famosa visionaria era riccamente dotata di quel che gli uomini medievali definivano doni di natura e fortuna, come anche grazia.

Ildegarda nacque presso Magonza da una nobile famiglia di Bermersheim: suo padre si chiamava Ildeberto e la madre Matilde, come sappiamo da Goffredo, monaco di Disibodenberg, che compose il I libro di una Vita sanctae Hildegardis (poi proseguita da Teodorico di Echternach, che aggiunse un II e un III libro e la Prefazione). All'età di 8 anni fu offerta a Dio come oblata e crebbe nell'eremo di Giuditta di Sponheim, vicino al fiorente monastero di San Disibodo, dove prese i voti monacali tra gli anni 1112 e 1115. L'eremo nel frattempo si stava trasformando in un convento, nel quale si osservava la regola benedettina e, quando Giuditta morì nel 1136, le monache elessero come badessa (magistra) Ildegarda.

Cinque anni dopo, all'età di 43 anni, ella si sentì investita da una missione profetica, ma intraprese l'importante carriera pubblica e letteraria, solo dopo aver ricevuto l'incoraggiamento di san Bernardo; tale carriera era destinata a durare per quasi quarant'anni. Alla sua morte, all'età di 81 anni, aveva trovato il tempo e la forza per fondare due monasteri, uno sul Rupertsberg, vicino a Bingen, l'altro a Eibingen, sull'altra riva del Reno e per intraprendere quattro visite di prolungata predicazione tra il 1161 e il 1163, rispettivamente in Svevia e lungo il Reno fino a Werden, e per dare consigli a una fiumana senza fine di visitatori e pellegrini alla fine degli anni cinquanta lungo il Meno, da Magonza a Bamberg; poi nel 1160 da Treviri, dove tenne una predica memorabile contro il clero corrotto della città, risalendo il corso della Mosella fino a Metz. Nel frattempo componeva tre importanti opere teologiche, un'enciclopedia scien-tifica e medica, un ciclo di canti liturgici, due vite di santi (la prima è la Vita sancti Disibodi composta nel 1170, la seconda è la Vita di san Ruperto) e una quantità assai notevole di lettere.
Non è facile accettare l'immagine che ella fornisce di sé come di una povera donna debole e fragile fin dall'infanzia; memori dell'esempio di Paolo e della necessità $\mathrm{d}$ essere (o almeno di apparire) umili, gli scrittori monastici durante il Medioevo reclamizzavano le proprie manchevolezze, sia di saggezza o di santità, sia di istruzione o di stile. Pertanto quando santa Ildegarda nelle sue prefazioni ricorda ai lettori la sua fragilità fisica, la sua scarsa educazione e il suo rozzo latino, sta collocandosi in una lunga serie di monaci retoricamente umili. Inoltre le sue proteste comportano più di un semplice topos di umiltà, paradossalmente però ella sta dicendo la verità: la sua salute era davvero precaria, i suoi studi scolastici erano scarsi (non però la sua cultura), la sua prosa incolta e rozza. A maggior ragione, però, Ildegarda avrebbe potuto lamentare una 'debolezza' più autentica di quella di qualsiasi dei suoi compagni teologi, per un motivo molto semplice: essere donna nella Chiesa del secolo XII significava, tra l'altro, essere folle, debole, umile e disprezzata agli occhi del mondo. Essere poi una predicatrice itinerante poteva significare solamente due cose: follia eretica, o potere divino, reso perfetto nella debolezza ${ }^{1}$

Accanto alla poesia mistica Ildegarda affronta anche altri campi del sapere: cosmologia, etica e medicina al punto che Peter Dronke (1986: 195) la paragona a Goethe.

In questi scritti Ildegarda si dimostra capace (benché fosse priva di qualsiasi cultura perché era stata educata da una donna ignorante) di comprendere i testi dei profeti, i Vangeli ecc. ecc: e di illustrarli componendo inoltre canzoni e inni in lode di Dio e dei santi, ancorché priva di una benché minima cultura musicale. Quando i testi di queste visioni furono portati a Treviri a papa Eugenio III, egli li lesse e li fece leggere, inviando a Ildegarda una lettera di benedizione. In grazia di questo dono Ildegarda assunse il ruolo di profetessa e fu poi soprannominata "la sibilla del Reno".

Ancora Peter Dronke osserva: "Ildegarda usa visio per designare tre cose correlate: la sua particolare facoltà o capacità di avere visioni; l'esperienza di questa facoltà e il contenuto dell'esperienza, tutto ciò che vede nella sua visio. Il modo della sua visione è del tutto inconsueto: vede 'nell'anima' mentre gode di tutti i poteri della normale percezione, di cui resta pienamente padrona" (1986: 197).

RiccardodiSan Vittore, mistico scozzese contemporaneo di Ildegarda, nel ‘Commento all'Apocalisse' codifica l'esistenza di quattro tipi di visione: due esterni: fisici e due interni: spirituali. Dei due modi di visione spirituale, uno è quello degli occhi del cuore, quando l'anima umana, illuminata dallo Spirito Santo, viene condotta, attraverso le apparenze delle cose visibili, e attraverso le immagini presentate come figure e segni,

1 Per quel che riguarda l'epoca tormentata in cui si trovò a vivere Ildegarda, un'epoca contrassegnata dalla prolungata lotta tra la Chiesa e l'Impero e dalle prime crociate, a favore della seconda delle quali aveva predicato san Bernardo a Vézelay nel 1146, cfr. G.Epiney-Burgard - E. Zum Brunn Femmes troubadours de Dieu, Turnhout 1988, p.27. 
alla conoscenza delle cose invisibili. Questo è quel che Dionigi aveva chiamato visione simbolica. La seconda, che Dionigi aveva chiamato visione anagogica, avviene quando l'anima umana, attraverso un'aspirazione interiore, si leva alla contemplazione delle cose celesti senza la mediazione di alcuna figura visibile.

Le visiones di Ildegarda appartengono piuttosto al terzo tipo della classificazione di Riccardo, perché la badessa è illuminata dalla voce spirituale che ode nella visio e le spiega il significato figurale o allegorico. La particolarità che distingue le visioni di Ildegarda sta nel fatto che contemporaneamente funziona anche la sua vista fisiologica: non si tratta quindi né di sogno, né di sogno a occhi aperti, né di trance, né di allucinazione, né di extasis.

Insieme alla visio si manifesta però la malattia che la sfianca. Come Rosvita, Ildegarda appare morbosamente intimidita dal mondo maschile che la circonda e fino a quando questo mondo 'maggiore' non le accetta, esse hanno la tentazione di nascondere, dissimulare o addirittura abbandonare il proprio talento. Per entrambe il primo motivo che le rende più sicure è l'amicizia con un'insegnante, in entrambi i casi una badessa di nobile nascita: Gerberga per Rosvita, Giuditta di Sponheim per Ildegarda.È grazie a loro, infatti, che gli uomini vengono informati delle rispettive qualità delle loro pupille. La pressura di Ildegarda a rivelare le proprie visioni si accompagnava a un senso di energia, che ella non aveva mai conosciuto nei lunghi anni di indisposizione. Volmer, il monaco di cui ella si fidava, fu lieto di diventarne aiutante e segretario.

Nel 1136 Ildegarda, come abbiamo già ricordato, fu eletta badessa dalle monache di Disimbodenberg, succedendo all'amata maestra Giuditta di Sponheim. Fu così che tra il 1137 e il 1147 il potente mondo maschile cominciò ad accettare progressivamente le sue eccezionali qualità: dapprima nell'arcivescovato di Magonza, poi nella curia papale. A partire dal 1147 Ildegarda, date ormai per sicure le sue doti profetiche, viene spesso sollecitata a dare consigli alle più importanti autorità secolari e religiose del suo tempo: i sovrani Corrado III, Federico Barbarossa (che cercò invano di dissuadere dapprima nel 1160 e poi nel 1177 dallo scisma), Enrico II d'Inghilterra, Eleonora d'Aquitania e l'imperatrice di Bisanzio, Irene, e i papi Anastasio IV, Adriano IV, nonché, naturalmente, Eugenio III. Nel 1151 si trovò ad affrontare una prova durissima perché, incitata dalla madre, marchesa von Stade (donna di ambizione smisurata) Riccarda, la sua allieva prediletta, manifestò aperti segni di indipendenza e, insieme con Adelaide (che della marchesa era la nipote) affermò che desiderava lasciare il Rupertsberg.

A questo punto Ildegarda, sentendosi tradita, scrisse alla marchesa una lettera molto accorata: "Vi supplico, vi prego di non turbare la mia anima così profondamente, tanto da portare lacrime amare ai miei occhi e ferire il mio cuore con ferite atroci per via delle amatissime mie figlie Riccarda e Adelaide. Le vedo fulgide nell'alba, adornate di perle di virtù. Quindi abbiate cura perché non avvenga che per vostro volere, avviso e connivenza, i loro sensi e le loro anime siano stornate dalla sublimità della grazia. Giacché la posizione di badessa, che voi desiderate per loro, è certo, certo, certo non compatibile con Dio, o con la salvezza delle loro anime" (Dronke, 1986: 206). Poi, presa da una furia scatenata, se la prende con tutto e con tutti: con l'arcivescovo di Magonza, con la stessa Riccarda (in una lettera a suo fratello) e, soprattutto con l'abate Cunone; ma, comportandosi così, diede spettacolo di sé, come non aveva fatto mai (pur temendolo) nel caso delle precedenti visioni.

Poi tuttavia tentò di rassegnarsi e scrisse a Riccarda questa lettera: “Figlia, ascolta me, tua madre in spirito, che ti dico: Il mio dolore aumenta. Il dolore uccide la grande fiducia, il grande conforto che trovai in un essere umano. D'ora in avanti dirò: 'È bene riporre le proprie speranze nel Signore piuttosto che riporle nei potenti del mondo'. Vale a dire che si deve mirare a quell'Uno che è in alto, il vivente, senza l'offuscamento di alcuno degli amori o delle flebili fedi che per qualche tempo può offrirci la buia regione sublunare. Colui che contempla Dio eleva così gli occhi come un'aquila verso il sole. E perciò non bisogna guardare le figure eminenti, che cadono come cadono i fiori. Non sono riuscita a fare questo, perché ho amato una persona nobile. Ora ti dico: ogniqualvolta ho peccato così, Dio mi ha reso chiaro il peccato con l'esperienza dell'angoscia o della sofferenza: e questo ora è accaduto con te, come sai tu stessa. Ora nuovamente dico: Ahimè, madre, ahimè, figlia. Perché mi hai abbandonata come un'orfana? Amavo la nobiltà del tuo comportamento, la tua saggezza e castità, la tua anima, tutta la tua vita, tanto che molti dicevano: che stai facendo? Ora, tutti coloro che provano un dolore come il mio piangano con me, tutti coloro che mai, per l'amore di Dio, hanno provato in cuor loro e nell'animo un amore profondo per un essere umano come io per te, per una persona strappata loro in un attimo, come tu sei stata strappata a me. Ma possa precederti l'angelo di Dio, il figlio di Dio proteggerti, sua madre difenderti. Ricorda la tua povera madre Ildegarda, che non venga meno la tua felicità" (Dronke, 1986: 208-9).

Ma torniamo adesso all'importanza delle Crociate per la vita della santa: quando Ildegarda nacque, i primi crociati erano in marcia verso Gerusalemme. “Nel 1095 il papa Urbano II aveva incitato la cristianità a combattere contro l'Islam e a riconquistare dalle mani dei Turchi il Santo Sepolcro. La sua predica trovò un'eco fanatica: decine di migliaia di uomini di ogni strato sociale lasciarono senza un attimo di esitazione ogni loro avere, abbandonarono moglie e figli per lanciarsi nell'avventurosa impresa. Si posero sulle spalle una pesante croce di legno o si cucirono simbolicamente una rossa croce di stoffa sul dorso e partirono. Nel 1099 Goffredo di Buglione conquistò Gerusalemme. Le Crociate successive non ottennero più alcun successo; un secolo e mezzo più tardi i luoghi santi furono persi per sempre" (Breindl, 1989: 14-15). Ma 
accanto al fanatismo dei crociati esisteva anche chi viveva nel silenzio e nella preghiera lontano dal mondo: erano gli eremiti o 'murati vivi'.

Questa era stata appunto la scelta di vita di Giuditta di Sponheim, a cui i genitori di Ildegonda, come abbiamo ricordato sopra, avevano affidato la custodia della loro ultimogenita. Giuditta aveva già un'altra piccola allieva che era una sua lontana parente. Il $1^{\circ}$ novembre 1106 Giuditta, contessa giovane e bella, salì con le due bimbe il monte del convento (il Disibodenberg appunto) guidando una lunghissima processione di amici e parenti. Al loro ingresso i monaci intonarono salmi e canti nuziali: poi cominciò la cerimonia secondo il rito sepolcrale, perché per Giuditta da quel momento la vita sarebbe diventata un continuo morire per Cristo per potere un giorno risorgere con lui. Terminata la messa, i monaci murarono la porta alle spalle di Giuditta e delle due bimbe e così per loro fu chiuso ogni collegamento col mondo esterno. Tra gli insegnamenti di Giuditta, Ildegarda era affascinata soprattutto dal canto e manifestava cantando le sue eccezionali doti musicali, che costituivano l'orgoglio di Giuditta. Ma, accanto a Giuditta, Ildegarda aveva come maestro Volmer, il monaco che l'abate Cunone aveva incaricato di fungere da assistente spirituale per le murate vive.

Mentre l'esempio di Giuditta trovava seguito nel paese, per cui, col passare degli anni, si formò una piccola comunità di 12 donne, le visioni di Ildegarda non erano cessate e quando a 15 anni comprese che era la sola ad averle, non disse più nulla su quello che le capitava. Ma, come abbiamo già ricordato, all'età di 42 anni e 7 mesi "si manifestò una luce ignea abbagliante, che venendo dal cielo che si era aperto, infiammò completamente il mio cervello e come una fiamma che non brucia ma riscalda dette fuoco completamente al mio cuore e al mio petto. E immediatamente diventai sapiente nell'interpretazione dei libri sacri, cioè il Salterio, il Vangelo e gli altri volum dell'Antico e del Nuovo Testamento" (Flanagan, 1991: 14). Ciononostante Ildegarda esitava a eseguire l'ordine divino, temendo di essere impari al compito, come ci spiega con queste parole: 'Ma io benché vedessi e udissi queste cose, tuttavia per l'incertezza, le opinioni sbagliate, i diversi discorsi degli uomini, non comunque per ostinazione, ma per umiltà, mi rifiutavo di scrivere, finché non caddi a letto malata, abbattuta dal castigo divino'.

Quando l'allieva prediletta Riccarda entrò nella sua cella e le chiese cosa fosse successo, Ildegarda le confidò l'ordine ricevuto da Dio. Allora Riccarda le consigliò di consultarsi con Volmer; Ildegarda, già più rilassata, raccontò la sua visione all'assistente spirituale, che la conforto assicurandole che, se non si sentiva in grado di affrontare da sola il compito che Dio le aveva affidato, qualcuno l'avrebbe certamente aiutata. Ildegarda, a questo punto gli chiese se avrebbe accettato di assumersi proprio lui questo incarico; ottenuto il permesso dell'abate Cunone, fu incoraggiata a incominciare a scrivere le visioni che stanno alla base dello Scivias e "ripresami dalla malattia, in meno di dieci anni portai a termine quest'opera".

Ma torniamo a parlare del monastero di Disibodenberg: esso era un monastero doppio, in una parte del quale vivevano i monaci, sotto la guida dell'abate Cunone, mentre in un'altra vivevano le monache guidate dalla badessa Ildegarda. Come abbiamo ricordato, però, Ildegarda "fu sempre una creatura doppia: una debole e inferma dimora femminile, e un essere privilegiato attraverso cui parlava lo Spirito Santo, una visionaria e una profetessa" "(Guiducci, 1990: 114).

La Guiducci continua poi così: "Fra le profezie che Ildegarda pronunciò ci fu quella sulla 'quinta epoca' o XVI secolo, nella quale l'Impero si sarebbe disintegrato e si sarebbe scavato uno scisma (allusione allo scisma della Chiesa d'Inghilterra)" e "ogni paese e ogni popolo si sceglieranno un loro re. Il papa decadrà dalla sua antica autorità a un tal punto da potere a malapena conservare sotto la sua tiara Roma e qualche tratto d'attorno...molti uomini torneranno ai costumi degli antichi, e i conventi saranno soppressi" (Guiducci, 1990: 116). La studiosa ricorda ancora che per curare Sigewize, una giovane nobildonna posseduta dal demonio, che, attraverso la sua voce, aveva reclamato la Gran Vecchia che viveva nell'alto Reno (cioè Ildegarda) ella mise in scena qualcosa di simile al dialogo teatrale da lei composto con il titolo Ordo virturum. Nel campo della medicina compose il trattato Causae et curae, che faceva parte di una più ampia sintesi intitolata Subtilitates naturarum diversarum creaturarum. Mentre si trovava nel monastero di Rupertsberg, una visione le ordinò di comporre una seconda opera dopo lo Scivias e la santa scrisse, fra il 1158 e il 1163, il Liber vitae meritorum che si occupa dei vizi e delle virtù degli uomini. Poi una nuova visione - tre torri allegoriche che nascondevano alla vista un edificio enigmatico - la indusse a scrivere il suo capolavoro, ovvero il Liber divinorum operum, in cui ricorre una concezione positiva dell'uomo, come manifestazione di Dio onnipotente. Fra le opere minori di ardua collocazione cronologica, vanno poi collocate la Lingua ignota, ovvero "Il linguaggio segreto", costituito da un elenco di parole corrispondente a una lista eclettica di nomi, e le Litterae ignotae, una sorta di alfabeto alternativo usato come codice segreto in ambiente domestico.

Per concludere vorrei ricordare che il processo di canonizzazione iniziato nel XII secolo non andò a buon fine, ma nel secolo seguente fecero la loro apparizione nei martirologi il suo nome e la sua festa e, infine, che esso comparve in un'indulgenza concessa dal papa Giovanni XXII ad Avignone nel 1324. La certificazione definitiva della sua posizione di santa ci fu soltanto quando il suo nome fu incluso nel "Martirologio Romano" di Cesare Baronio nel corso del XVI secolo. 


\section{RIFERIMENTI BIBLIOGRAFICI:}

Breindl, E., L'erborista di Dio. Santa Ildegonda mistica medievale (trad.it. di G. Lupi), Milano (ed originale Das große Gesundheitsbuch der Hl.Hildegard von Bingen, Aschaffenburg, 1983), 1989.

Dronke P., Donne e cultura nel Medioevo, (trad. it E. Randi), Milano, (ed.orig. Women Writers of the Middle Ages, Cambridge 1984), 1986.

Epiney-Burgard, G, Zum Brunn, E., Femmes troubadours de Dieu, Turnhout, 1988.

Flanagan, S., Ildegarda di Bingen. Vita di una profetessa,Firenze (trad.it M. Pereira), Ildegarda di Bingen. Vita di una profetessa,Firenze, (ed. Orig. Hildegard of Bingen, 1098-1179. A visionary life, London and New York 1989), 1991.

F?hrk?tter, A., Hildegard Von Bingen: Briefwefschel (Letters), Otto Muller, Vertag, Salzburg, 1990.

Newmann, B., Sister of Wisdom. St.Hildegard's Theology of the Feminine Berkeley, Los Angeles, California, 1987. 